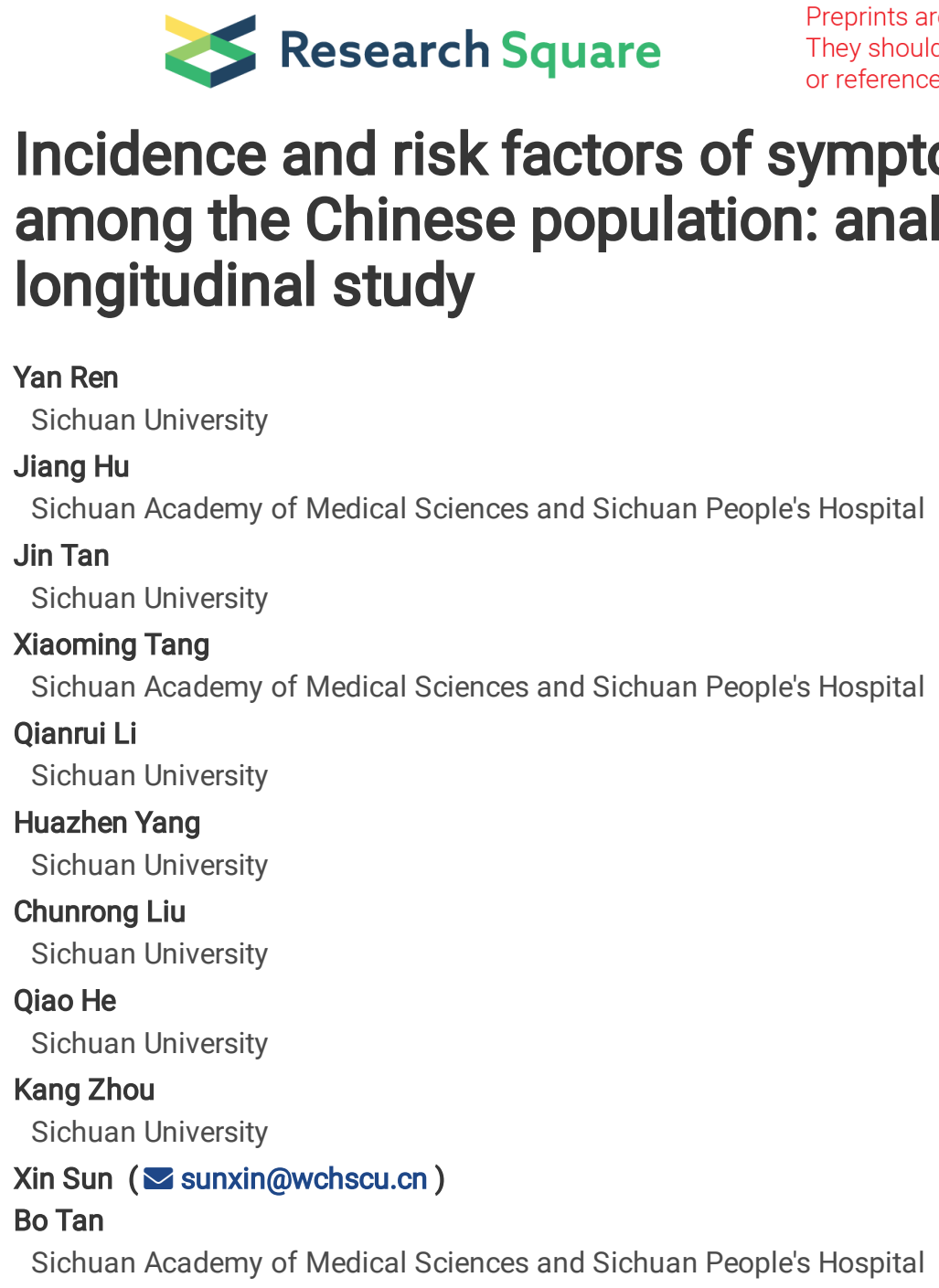

\title{
Incidence and risk factors of symptomatic knee osteoarthritis among the Chinese population: analysis from a nationwide longitudinal study
}




\section{Abstract}

Background: To investigate the incidence of symptomatic knee osteoarthritis (OA) and identify risk factors among the Chinese population. Methods: The China Health and Retirement Longitudinal Study (CHARLS) is a nationwide longitudinal survey of persons aged $\geq 45$ years. The baseline survey for the CHARLS study was conducted in 2011 using a four-stage, stratified, cluster probability sampling design. The respondents were then followed up every 2 years through a face-to-face interview. Using the national survey data collected from the CHARLS, we estimated the incidence of symptomatic knee OA, taking into account the complex survey design and response rate. We applied weighted logistic regression analysis to identify risk factors. Results: In the 4 years of follow-up, the cumulative incidence of symptomatic knee OA among middle-aged and older Chinese adults was $8.5 \%$; the incidence was higher among females (11.2\%) than males (5.6\%). In the longitudinal analysis, female, rural area, West region, low education level, lack of physical activities, and the presence of heart disease, kidney disease, and digestive disease at the baseline survey were associated with a higher risk of symptomatic knee OA. The risk in persons doing regular physical activities was $53 \%$ lower than that in persons who were not active (odds ratio (OR) 0.47 [95\% $\mathrm{Cl}$ ) $0.29-0.76]$ ). Histories of heart disease (odds ratio (OR) 1.40 [95\% confidence interval (Cl) 1.07-1.82]), kidney disease (OR 1.80 [95\% Cl 1.35-2.39]), and digestive disease (OR 1.54 [95\% $\mathrm{Cl} 1.30-1.82])$ were associated with a higher risk of symptomatic knee OA. Conclusion: The cumulative incidence of symptomatic knee OA over 4 years was relatively high, and varied by province and region. Lack of physical activities, and the presence of heart disease, kidney disease, and digestive disease were associated with a higher risk of symptomatic knee OA.

\section{Background}

China has the world's largest elderly population, and the whole nation is aging fast - the proportion of aging population ( $\geq 60$ years) was estimated to be $14.9 \%$ in 2013, 25.3\% in 2030, and over 30\% in 2050 [1]. Rapid ageing has led to an increasing healthcare burden attributable to age-related diseases [2]. Knee osteoarthritis (OA) is a common disease condition associated with becoming older. It is one of the leading causes of disabilities, that primarily affect elderly adults [3, 4]. The disease can substantially reduce quality of life [5]; severe cases may even lead to knee-joint replacement. The costs for knee OA management are usually very high, placing a heavy burden upon families and society [6-8].

Epidemiological studies to understand the burden of knee OA are currently of great importance for health-care policy makers and clinicians. A previous study using a nationwide survey found that the prevalence of symptomatic knee OA among Chinese adults aged $\geq$ 45 years was $8.1 \%$ and increased with age [9], and concluded that future studies are needed to identify the risk factors for symptomatic knee OA. To date, however, no data are available on the incidence of symptomatic OA among the middle-aged and older Chinese population. Additionally, due to significant imbalances in socioeconomic development, environmental conditions, lifestyle patterns, and health-care utilization among different geographic regions in China, large variations in the incidence of symptomatic knee OA may be present among these populations. The lack of information may hinder the effective planning and execution of health-care strategies, as well as efficient use of health care sources.

In addition, understanding the risk factors for knee $\mathrm{OA}$ is important for managing health among adults, particularly for elderly populations. Several longitudinal studies conducted in the U.S.A., the U.K., Japan, and Europe have investigated risk factors for the development of symptomatic knee OA [10-13]. However, these studies were conducted in developed countries. The findings from them have limited implications for Chinese populations, because socioeconomic status, environmental factors, and lifestyle patterns differ substantially between the developing countries and the developed world. To date, no longitudinal studies have been conducted to examine risk factors for knee OA among the elderly Chinese population. Only a few cross-sectional studies investigated factors associated with knee OA [9, 14-17].

In order to bridge this important evidence gap, we conducted a cohort study analysis, using data from the China Health and Retirement Longitudinal Study (CHARLS), to investigate risk factors for the development of symptomatic knee OA in Chinese adults aged 45 years or older. In addition, we determined the incidence of symptomatic knee OA for this population.

\section{Methods}

Data sources

The data sources of our study came from the CHARLS, a nationwide longitudinal survey among Chinese adults aged $\geq 45$ years. A detailed description of the methodology was reported previously [18]. In brief, the CHARLS study employed a four-stage probability sampling approach to select representative samples of eligible participants. Specifically, the first stage involved a random selection of 150 
counties from 28 provinces of China given a probability-proportional-to-size (PPS) from a sampling frame containing all county-level units with the exception of Tibet. The sample was stratified by region and within region by urban or rural status and gross domestic product (GDP) per capita. The second stage randomly selected administrative villages (cun) in rural areas and neighborhoods (shequ) in urban areas, as primary sampling units (PSUs), for which three PSUs were selected from each county. In the third stage, a random sample of 24 households was selected on the basis of geographic locations and lists of each PSU. Finally, a resident aged $\geq 45$ years was randomly selected from a household, and an interview was undertaken with the selected resident and their spouse. Taking into account of the complex survey design and the non-response rate for the CHARLS, the weighted value was constructed from the sampling probability and response probability, and was provided by the CHARLS database.

In the CHARLS, the baseline survey was conducted in 2011, and 17,708 respondents were interviewed from 150 representative counties of 28 provinces across China. Using structured questionnaires, data were collected regarding demographic information, health status (e.g. self-reported general health, doctor-diagnosed chronic and infectious disease, lifestyle and life behavior, including sleep and physical activity), socioeconomic status and biomedical measurements (e.g. blood pressure, pulse, peak expiratory flow, height, weight, waist size). The respondents were followed up every 2 years through a face-to-face interview.

The current study is a secondary analysis of the CHARLS public data. All data collected in the CHARLS are maintained at the National School of Development of Peking University, Beijing, China. The datasets are available from http://charls.pku.edu.cn/pages/data/111/zh$\mathrm{cn}$.html. The CHARLS was approved by the Ethical Review Committee of Peking University, and all participants signed informed consent at the time of participation. No separate ethical approval was required for our study.

Study population and outcome measurement

In our study, the outcome of interest was a reported symptomatic knee OA. We included all the participants in this study who were free from symptomatic knee OA at the baseline survey. The outcome symptomatic knee OA was ascertained if a participant responded with "yes" to the first two of the following questions, and responded "the knees" to the third question. (1) Have you been diagnosed with arthritis or rheumatism by a doctor? (2) Are you often troubled with any body pain? If the participant responded "yes" to the second question, they were presented with question (3): On what part of your body do you feel pain? (list all body pains) (Supplementary table 1). Our definition of symptomatic knee OA was consistent with one published study [9], who used the same CHARLS database.

Covariates

The data for our study included demographic information (gender, age, area of residence, and region), socioeconomic status (education), health status (some diseases, has undertaken some physical activities), and anthropometric measurements (height and weight). All the information was collected from reporting by the participants.

The covariates were gender, age, area, region of residence within the country, education, body mass index (BMI), having undertaken physical activities in the last month, and history of hypertension, dyslipidemia, diabetes, chronic lung disease, liver disease, heart disease, stroke, kidney disease, digestive disease, psychiatric disease, or asthma. The body mass index (BMI) was calculated as the individual's weight divided by the square of their height $\left(\mathrm{kg} / \mathrm{m}^{2}\right)$. A person doing physical activities (e.g. dancing, body building) or not in the last month was categorized as "yes" or "no". A person with a self-reported history of health conditions or without at the baseline survey was categorized as "yes" or "no".

Statistical analysis

We categorized the following variables, including age ( $\$ 50$ years, 50-59 years, 60-69 years, and $\geq 70$ years), area (urban vs. rural), region of residence within the country (East vs. Central vs. West), education (no formal education vs. elementary school vs. middle school vs. high school or higher), and BMI by World Health Organization (WHO) criteria (underweight, normal, and overweight: $<18.5,18.5-24.9$, and $\left.\geq 25.0 \mathrm{~kg} / \mathrm{m}^{2}\right)$. Because of a limited number in the obesity group $\left(\mathrm{BMI} \geq 30.0 \mathrm{~kg} / \mathrm{m}^{2}\right)$, we combined obese and overweight participants.

Taking into account the complex survey design and the non-response rate for the CHARLS survey, we used the inverse probability weighting method (the Proc Surveyfreq procedure in SAS version 9.4) to calculate the weighted cumulative incidence of symptomatic knee $\mathrm{OA}$ and the weighted percentage of symptomatic knee OA. Then we used the Taylor linearized method to estimate the variance of weighted cumulative incidence. According to its variance, we calculated its $95 \%$ confidence interval (Cl).

We used the Proc Surveylogistic procedure in SAS version 9.4 to examine the risk factors of symptomatic knee OA. The basic model can be written as follows: 


$$
\begin{aligned}
& \operatorname{logit}(\pi)=\log \left(\frac{\pi}{1-\pi}\right)=\beta_{0}+\sum \beta_{i} x_{i} \quad \text { (Model1) } \\
& \operatorname{logit}(\pi)=\log \left(\frac{\pi}{1-\pi}\right)=\beta_{0}+\sum \beta_{i} x_{i}+\sum \beta_{j} \text { diseases }_{j} \quad(\text { Model2) }
\end{aligned}
$$

The Surveylogistic procedure fits linear logistic regression models for discrete response survey data by the method of maximum-likelihood method. For statistical inferences, Proc Surveylogistic incorporates complex survey sample designs, including designs with stratification, clustering, and unequal weighting. In model 1, we adjusted for some potential confounders, including gender, age, area, region, education, BMI group, doing physical activities in the last month. In model 2, we further added other potential confounders, including histories of hypertension, dyslipidemia, diabetes, chronic lung disease, liver disease, heart disease, stroke, kidney disease, digestive disease, psychiatric disease, and asthma. Odds ratios (ORs) and 95\% Cls were presented for variables in the models. We used a complete case analysis for the primary risk factors analysis. With respect to missing data, we used the multiple imputation method, and compared the differences of missing data in demographic and clinical variables between the case and control group; then, we performed sensitivity analyses to estimate risk factors of symptomatic knee OA.

\section{Results}

In the CHARLS, 15,910 respondents were free from the symptomatic knee OA at the 2011 national baseline survey, among whom 2833 were lost to follow-up in 2013 and 2015. As a result, 13,077 remained in the cohort until 2015 and were finally used in our study (Fig. 1).

Among the 13,077 respondents included in this analysis (Table 1): 6726 (51.5\%) were women; the mean age was 64.2 years (SE 0.09); $8140(62.3 \%)$ lived in rural areas. The respondents were generally equally distributed across geographic regions $(36.4 \%, 32.4 \%$, and $31.2 \%$ from East, Central, and West of China, respectively). Among all respondents, $40.0 \%$ completed elementary school only, and $12.0 \%$ received high school or higher education; $62.9 \%$ had normal weight (BMI: $\left.18.5-24.9 \mathrm{~kg} / \mathrm{m}^{2}\right), 6.4 \%$ were underweight (BMl: $<18.5 \mathrm{~kg} / \mathrm{m}^{2}$ ), and $30.7 \%$ were overweight (BMI: $\geq 25.0 \mathrm{~kg} / \mathrm{m}^{2}$ ). Most respondents did not have pre-specified disease conditions at the baseline survey, as per physician diagnosis. 
Table 1

Characteristics of respondents by status of symptomatic knee OA

\begin{tabular}{|c|c|c|c|c|c|c|c|c|c|}
\hline \multirow[t]{2}{*}{ Variables } & \multicolumn{3}{|c|}{ All respondents } & \multicolumn{3}{|c|}{ Symptomatic knee OA } & \multicolumn{3}{|c|}{ Without symptomatic knee OA } \\
\hline & $\begin{array}{l}\text { No. (n } \\
= \\
13077 \text { ) }\end{array}$ & $\begin{array}{l}\% \\
\text { unweighted }\end{array}$ & $\begin{array}{l}\% \\
\text { weighted }\end{array}$ & $\begin{array}{l}\text { No. } \\
(n= \\
1196)\end{array}$ & $\begin{array}{l}\% \\
\text { unweighted }\end{array}$ & $\begin{array}{l}\% \\
\text { weighted }\end{array}$ & $\begin{array}{l}\text { No. (n } \\
= \\
11881 \text { ) }\end{array}$ & $\begin{array}{l}\% \\
\text { unweighted }\end{array}$ & $\begin{array}{l}\% \\
\text { weighted }\end{array}$ \\
\hline \multicolumn{10}{|l|}{ Gender } \\
\hline Male & 6347 & 48.55 & 48.15 & 396 & 33.11 & 31.71 & 5951 & 50.11 & 50.11 \\
\hline Female & 6726 & 51.45 & 51.85 & 800 & 66.89 & 68.29 & 5926 & 49.89 & 49.89 \\
\hline $\begin{array}{l}\text { Age, years } \\
(\text { mean } \pm \text { SE })\end{array}$ & 13077 & $\begin{array}{l}64.24 \pm \\
0.09\end{array}$ & $\begin{array}{l}64.45 \pm \\
0.13\end{array}$ & 1196 & $\begin{array}{l}64.92 \pm \\
0.27\end{array}$ & $\begin{array}{l}64.75 \pm \\
0.32\end{array}$ & 11881 & $\begin{array}{l}64.17 \pm \\
0.09\end{array}$ & $\begin{array}{l}64.42 \pm \\
0.14\end{array}$ \\
\hline$<50$ & 908 & 6.94 & 7.33 & 83 & 6.94 & 6.81 & 825 & 6.94 & 7.38 \\
\hline $50-59$ & 4043 & 30.92 & 31.36 & 318 & 26.59 & 26.73 & 3725 & 31.35 & 31.79 \\
\hline $60-69$ & 4776 & 36.52 & 34.88 & 461 & 38.55 & 38.89 & 4315 & 36.32 & 34.51 \\
\hline$\geq 70$ & 3350 & 25.62 & 26.43 & 334 & 27.93 & 27.57 & 3016 & 25.39 & 26.32 \\
\hline \multicolumn{10}{|l|}{ Area } \\
\hline Urban & 8140 & 62.25 & 54.68 & 396 & 33.11 & 67.11 & 5951 & 50.11 & 61.42 \\
\hline Rural & 4937 & 37.75 & 45.32 & 800 & 66.89 & 32.89 & 5926 & 49.89 & 38.58 \\
\hline \multicolumn{10}{|l|}{ Region } \\
\hline Central & 4237 & 32.40 & 30.36 & 369 & 30.85 & 29.14 & 3868 & 32.56 & 30.47 \\
\hline East & 4760 & 36.40 & 39.94 & 270 & 22.58 & 24.53 & 4490 & 37.79 & 41.37 \\
\hline West & 4080 & 31.20 & 29.70 & 557 & 46.57 & 46.33 & 3523 & 29.65 & 28.16 \\
\hline \multicolumn{10}{|l|}{ Education } \\
\hline $\begin{array}{l}\text { No formal } \\
\text { education }\end{array}$ & 3441 & 26.31 & 25.23 & 430 & 35.95 & 34.96 & 3011 & 25.34 & 24.32 \\
\hline $\begin{array}{l}\text { Elementary } \\
\text { school }\end{array}$ & 5230 & 39.99 & 39.31 & 529 & 44.23 & 45.14 & 4701 & 39.57 & 38.78 \\
\hline $\begin{array}{l}\text { Middle } \\
\text { school }\end{array}$ & 2839 & 21.71 & 21.81 & 163 & 13.63 & 13.72 & 2676 & 22.52 & 22.56 \\
\hline $\begin{array}{l}\text { High school } \\
\text { or } \\
\text { Vocational } \\
\text { school or } \\
\text { higher }\end{array}$ & 1567 & 11.98 & 13.65 & 74 & 6.19 & 6.18 & 1493 & 12.57 & 14.34 \\
\hline \multicolumn{10}{|l|}{$\begin{array}{l}\text { BMI group } \\
\left(\mathrm{kg} / \mathrm{m}^{2}\right)\end{array}$} \\
\hline$<18.5$ & 678 & 6.45 & 6.30 & 72 & 7.32 & 6.82 & 606 & 6.36 & 6.25 \\
\hline $18.5-24.9$ & 6615 & 62.90 & 62.65 & 609 & 61.95 & 61.38 & 6006 & 63.00 & 62.78 \\
\hline$\geq 25.0$ & 3223 & 30.65 & 31.04 & 302 & 30.72 & 31.80 & 2921 & 30.64 & 30.97 \\
\hline \multicolumn{10}{|c|}{ Done some activities (such as played a sport, social, or other kind of club dancing, doing physical exercise, doing Qigong,et al. ) } \\
\hline Yes & 705 & 5.39 & 7.03 & 800 & 66.89 & 2.89 & 5926 & 49.89 & 5.66 \\
\hline No & 12372 & 94.61 & 92.97 & 396 & 33.11 & 97.11 & 5951 & 50.11 & 94.34 \\
\hline
\end{tabular}




\begin{tabular}{|c|c|c|c|c|c|c|c|c|c|}
\hline \multirow[t]{2}{*}{ Variables } & \multicolumn{3}{|c|}{ All respondents } & \multicolumn{3}{|c|}{ Symptomatic knee OA } & \multicolumn{3}{|c|}{ Without symptomatic knee OA } \\
\hline & $\begin{array}{l}\text { No. }(n \\
= \\
13077)\end{array}$ & $\begin{array}{l}\% \\
\text { unweighted }\end{array}$ & $\begin{array}{l}\% \\
\text { weighted }\end{array}$ & $\begin{array}{l}\text { No. } \\
(n= \\
1196)\end{array}$ & $\begin{array}{l}\% \\
\text { unweighted }\end{array}$ & $\begin{array}{l}\% \\
\text { weighted }\end{array}$ & $\begin{array}{l}\text { No. }(n \\
= \\
11881)\end{array}$ & $\begin{array}{l}\% \\
\text { unweighted }\end{array}$ & $\begin{array}{l}\% \\
\text { weighted }\end{array}$ \\
\hline Yes & 2930 & 22.52 & 23.26 & 800 & 66.89 & 27.17 & 5926 & 49.89 & 22.15 \\
\hline No & 10079 & 77.48 & 76.74 & 396 & 33.11 & 72.83 & 5951 & 50.11 & 77.85 \\
\hline \multicolumn{10}{|c|}{ Dyslipidemia } \\
\hline Yes & 1129 & 8.80 & 9.31 & 800 & 66.89 & 10.30 & 5926 & 49.89 & 8.74 \\
\hline No & 11704 & 91.20 & 90.69 & 396 & 33.11 & 89.70 & 5951 & 50.11 & 91.26 \\
\hline \multicolumn{10}{|l|}{ Diabetes } \\
\hline Yes & 670 & 5.17 & 5.51 & 800 & 66.89 & 7.06 & 5926 & 49.89 & 5.15 \\
\hline No & 12291 & 94.83 & 94.49 & 396 & 33.11 & 92.94 & 5951 & 50.11 & 94.85 \\
\hline \multicolumn{10}{|c|}{ Chronic lung } \\
\hline Yes & 1130 & 8.67 & 8.66 & 800 & 66.89 & 12.80 & 5926 & 49.89 & 8.29 \\
\hline No & 11901 & 91.33 & 91.34 & 396 & 33.11 & 87.20 & 5951 & 50.11 & 91.71 \\
\hline \multicolumn{10}{|c|}{ Liver disease } \\
\hline Yes & 434 & 3.34 & 3.39 & 800 & 66.89 & 4.74 & 5926 & 49.89 & 3.15 \\
\hline No & 12559 & 96.66 & 96.61 & 396 & 33.11 & 95.26 & 5951 & 50.11 & 96.85 \\
\hline \multicolumn{10}{|l|}{$\begin{array}{l}\text { Heart } \\
\text { disease }\end{array}$} \\
\hline Yes & 1335 & 10.27 & 10.10 & 800 & 66.89 & 16.81 & 5926 & 49.89 & 9.65 \\
\hline No & 11668 & 89.73 & 89.90 & 396 & 33.11 & 83.19 & 5951 & 50.11 & 90.35 \\
\hline \multicolumn{10}{|l|}{ Stroke } \\
\hline Yes & 237 & 1.82 & 1.99 & 800 & 66.89 & 2.85 & 5926 & 49.89 & 1.73 \\
\hline No & 12811 & 98.18 & 98.01 & 396 & 33.11 & 97.15 & 5951 & 50.11 & 98.27 \\
\hline \multicolumn{10}{|l|}{$\begin{array}{l}\text { Kidney } \\
\text { disease }\end{array}$} \\
\hline Yes & 671 & 5.16 & 5.29 & 800 & 66.89 & 8.88 & 5926 & 49.89 & 4.75 \\
\hline No & 12334 & 94.84 & 94.71 & 396 & 33.11 & 91.12 & 5951 & 50.11 & 95.25 \\
\hline \multicolumn{10}{|l|}{$\begin{array}{l}\text { Digestive } \\
\text { disease }\end{array}$} \\
\hline Yes & 2671 & 20.48 & 19.88 & 800 & 66.89 & 30.48 & 5926 & 49.89 & 19.29 \\
\hline No & 10374 & 79.52 & 80.12 & 396 & 33.11 & 69.52 & 5951 & 50.11 & 80.71 \\
\hline \multicolumn{10}{|c|}{$\begin{array}{l}\text { Psychiatric } \\
\text { disease }\end{array}$} \\
\hline Yes & 166 & 1.27 & 1.53 & 800 & 66.89 & 1.62 & 5926 & 49.89 & 1.22 \\
\hline No & 12860 & 98.73 & 98.47 & 396 & 33.11 & 98.38 & 5951 & 50.11 & 98.78 \\
\hline \multicolumn{10}{|l|}{ Asthma } \\
\hline Yes & 394 & 3.02 & 3.01 & 800 & 66.89 & 5.01 & 5926 & 49.89 & 2.86 \\
\hline No & 12636 & 96.98 & 96.99 & 396 & 33.11 & 94.99 & 5951 & 50.11 & 97.14 \\
\hline
\end{tabular}


In the 4 years of follow-up, 8.5\% (7.7-9.3\%) of participants developed symptomatic knee OA (Table 2). The cumulative incidence over 4 years was higher among females (11.2\%) than males (5.6\%). Respondents aged 60-69 years had the highest incidence, with $9.5 \%$ being affected with symptomatic knee OA. Respondents who resided in rural areas (10.4\%) had a greater cumulative incidence of symptomatic knee OA than those in urban areas (6.2\%). Those from the West (13.2\%) and Central regions (8.1\%) had a greater cumulative incidence of symptomatic knee OA than those from the East region (5.2\%). Cumulative incidence was much lower among respondents who had received a longer duration of education or undertook physical activities (e.g. dancing). The respondents who were affected by a physiciandiagnosed disease of other diseases at baseline survey had a greater cumulative incidence. 
Table 2

The cumulative incidence over 4 years of symptomatic knee OA by age, area, region, education, BMI group, activities and chronic disease (Values presented are the weighted incidence of symptomatic knee OA)

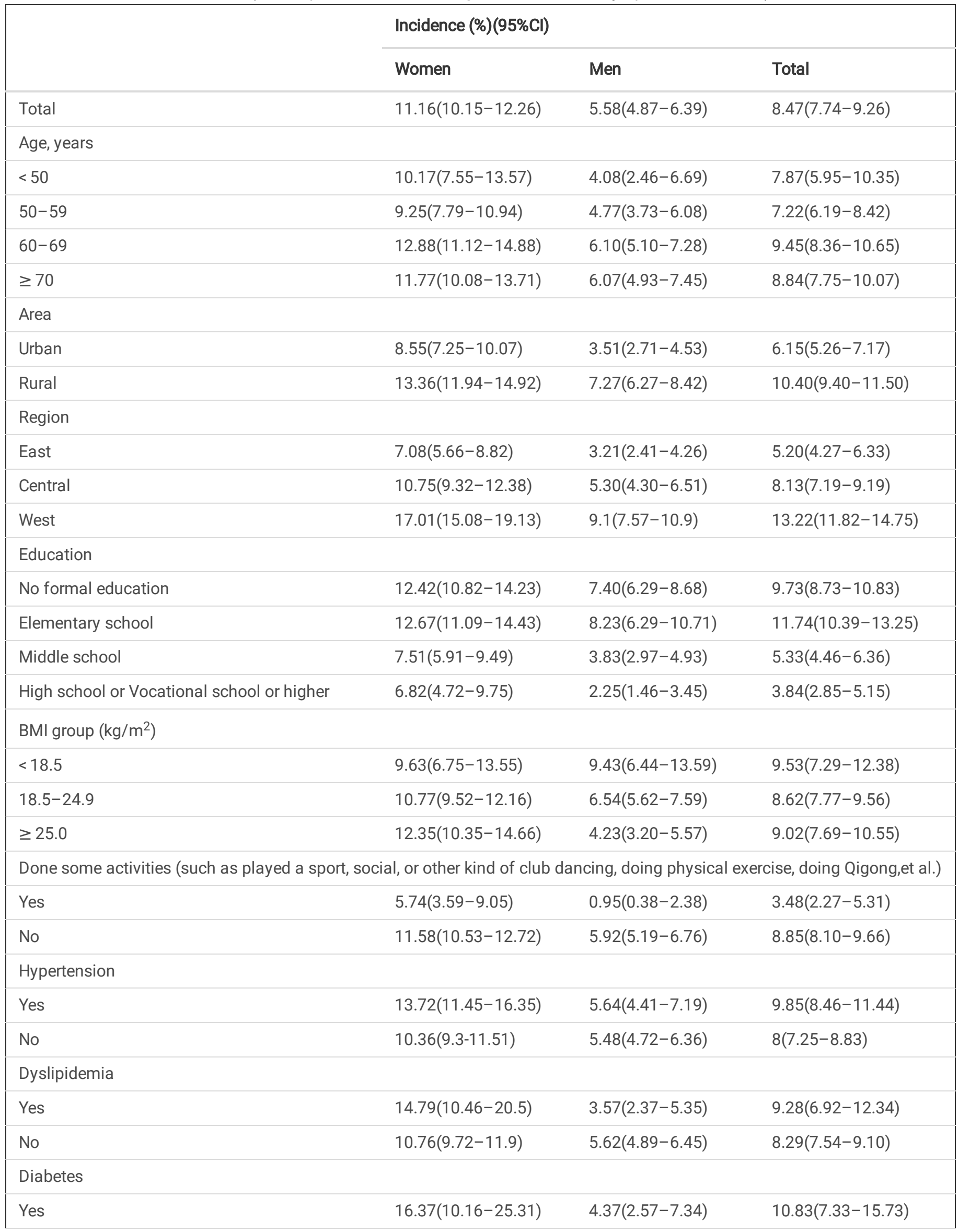




\begin{tabular}{|c|c|c|c|}
\hline & \multicolumn{3}{|l|}{ Incidence (\%)(95\%Cl) } \\
\hline & Women & Men & Total \\
\hline No & $10.87(9.84-11.99)$ & $5.59(4.87-6.42)$ & $8.31(7.57-9.12)$ \\
\hline \multicolumn{4}{|c|}{ Chronic lung disease } \\
\hline Yes & $17.61(14.13-21.74)$ & $9.00(6.70-11.98)$ & $12.51(10.40-14.98)$ \\
\hline No & $10.69(9.68-11.81)$ & $5.15(4.47-5.94)$ & $8.08(7.36-8.87)$ \\
\hline \multicolumn{4}{|c|}{ Liver disease } \\
\hline Yes & $15.31(10.59-21.62)$ & $9.09(6.06-13.43)$ & 11.78(8.74-15.69) \\
\hline No & $11.04(10.01-12.16)$ & $5.36(4.67-6.16)$ & $8.32(7.59-9.12)$ \\
\hline \multicolumn{4}{|c|}{ Heart disease } \\
\hline Yes & $19.03(16.12-22.32)$ & $6.94(4.83-9.86)$ & $14.02(12.00-16.32)$ \\
\hline No & $10.14(9.13-11.24)$ & $5.35(4.63-6.17)$ & $7.79(7.07-8.59)$ \\
\hline \multicolumn{4}{|c|}{ Stroke } \\
\hline Yes & $13.09(7.54-21.76)$ & $11.19(6.42-18.80)$ & $12.11(8.16-17.61)$ \\
\hline No & $11.14(10.12-12.24)$ & $5.47(4.76-6.29)$ & $8.41(7.69-9.20)$ \\
\hline \multicolumn{4}{|c|}{ Kidney disease } \\
\hline Yes & $18.52(13.48-24.9)$ & $10.82(7.75-14.93)$ & $14.19(11.33-17.64)$ \\
\hline No & $10.81(9.80-11.91)$ & $5.21(4.51-6.00)$ & $8.13(7.42-8.91)$ \\
\hline \multicolumn{4}{|c|}{ Digestive disease } \\
\hline Yes & $16.98(14.85-19.34)$ & $8.2(6.62-10.12)$ & $12.95(11.44-14.63)$ \\
\hline No & $9.63(8.58-10.80)$ & $4.92(4.18-5.77)$ & $7.33(6.61-8.13)$ \\
\hline \multicolumn{4}{|c|}{ Psychiatric disease } \\
\hline Yes & $11.74(5.9-22.00)$ & $1.71(0.22-12.09)$ & $8.99(4.93-15.82)$ \\
\hline No & $11.16(10.15-12.27)$ & $5.55(4.85-6.34)$ & $8.44(7.71-9.22)$ \\
\hline \multicolumn{4}{|c|}{ Asthma } \\
\hline Yes & $21.05(14.56-29.43)$ & $9.29(5.56-15.12)$ & $14.10(10.54-18.61)$ \\
\hline No & 10.93(9.91-12.04) & $5.44(4.73-6.25)$ & $8.30(7.57-9.09)$ \\
\hline
\end{tabular}

The cumulative incidence of symptomatic knee OA by province is presented in Fig. 2 . The six provinces with the lowest cumulative incidences (<5\%) were Beijing, Henan, Jiangsu, Liaoning, Guangdong, and Zhejiang. The three provinces with the highest cumulative incidences (>15\%) were Sichuan, Qinghai, and Yunnan.

Identification of risk factors

The multivariable weighted analyses showed that gender, area, region, education, not having undertaken any physical activities, and selfreported histories of heart disease, kidney disease, and digestive disease were significantly associated with symptomatic knee OA (Table 3). The risk of symptomatic knee OA was higher in women (adjusted OR 1.98, 95\% $\mathrm{Cl} 1.65-2.37$ ), rural areas (OR 1.32 [95\% Cl 1.08-1.60]), West (OR 2.33 [95\% Cl 1.89-2.87]) and Central regions (OR 1.49 [95\% Cl 1.19-1.87]), and in those with self-reported histories of heart diseases (OR 1.40 [95\% Cl 1.07-1.82]), kidney disease (OR 1.80 [95\% $\mathrm{Cl} 1.35-2.40]$ ), and digestive diseases (OR 1.54 [95\% Cl $1.30-1.82]$ ). In contrast, those with an education level of middle school (OR 0.69 [95\% Cl 0.52-0.90]), or high school or higher (OR 0.60 [95\% $\mathrm{Cl} 0.41-0.88]$ ), or who had undertaken some physical activities (OR $0.47,95 \% \mathrm{Cl} 0.29-0.76$ ) were associated with less likelihood of symptomatic knee OA. 
Table 3

Longitudinal analysis of symptomatic knee OA with different variables in different models

\begin{tabular}{|c|c|c|c|c|}
\hline \multirow[t]{2}{*}{ Variables } & \multicolumn{2}{|c|}{ Model 1} & \multicolumn{2}{|c|}{ Model 2} \\
\hline & OR & $95 \% \mathrm{Cl}$ & OR & $95 \% \mathrm{Cl}$ \\
\hline \multicolumn{5}{|l|}{ Gender } \\
\hline \multicolumn{5}{|l|}{ Male } \\
\hline Female & 1.97 & $1.65-2.37$ & 1.98 & $1.65-2.37$ \\
\hline \multicolumn{5}{|l|}{ Age, years } \\
\hline \multicolumn{5}{|l|}{$<50$} \\
\hline $50-59$ & 0.88 & $0.60-1.28$ & 0.88 & $0.60-1.29$ \\
\hline $60-69$ & 1.18 & $0.82-1.71$ & 1.14 & $0.78-1.64$ \\
\hline$\geq 70$ & 1.09 & $0.76-1.57$ & 1.01 & $0.70-1.47$ \\
\hline \multicolumn{5}{|l|}{ Area } \\
\hline \multicolumn{5}{|l|}{ Urban } \\
\hline Rural & 1.31 & $1.08-1.59$ & 1.32 & $1.08-1.60$ \\
\hline \multicolumn{5}{|l|}{ Region } \\
\hline \multicolumn{5}{|l|}{ East } \\
\hline Central & 1.56 & $1.24-1.97$ & 1.49 & $1.19-1.87$ \\
\hline West & 2.39 & $1.92-2.99$ & 2.33 & $1.89-2.87$ \\
\hline \multicolumn{5}{|l|}{ Education } \\
\hline \multicolumn{5}{|l|}{ No formal education } \\
\hline Elementary school & 1.14 & $0.93-1.38$ & 1.08 & $0.89-1.32$ \\
\hline Middle school & 0.70 & $0.54-0.92$ & 0.69 & $0.52-0.90$ \\
\hline High school or Vocational school or higher & 0.64 & $0.43-0.93$ & 0.60 & $0.41-0.88$ \\
\hline \multicolumn{5}{|l|}{ BMI group $\left(\mathrm{kg} / \mathrm{m}^{2}\right)$} \\
\hline \multicolumn{5}{|l|}{$<18.5$} \\
\hline $18.5-24.9$ & 1.08 & $0.79-1.47$ & 1.10 & $0.80-1.51$ \\
\hline$\geq 25.0$ & 1.25 & $0.89-1.75$ & 1.21 & $0.87-1.69$ \\
\hline \multicolumn{5}{|c|}{ Done some activities (such as played a sport, social, or other kind of club dancing, doing physical exercise, doing Qigong,et al. } \\
\hline \multicolumn{5}{|l|}{ No } \\
\hline Yes & 0.50 & $0.31-0.81$ & 0.47 & $0.29-0.76$ \\
\hline \multicolumn{5}{|l|}{ Chronic disease } \\
\hline Hypertension (Yes vs. No) & & & 1.12 & $0.92-1.37$ \\
\hline Dyslipidemia (Yes vs. No) & & & 1.07 & $0.75-1.52$ \\
\hline Diabetes (Yes vs. No) & & & 1.30 & $0.78-2.14$ \\
\hline Chronic lung disease (Yes vs. No) & & & 1.19 & $0.92-1.55$ \\
\hline Liver disease (Yes vs. No) & & & 1.22 & $0.83-1.80$ \\
\hline Heart disease (Yes vs. No) & & & 1.40 & $1.07-1.82$ \\
\hline
\end{tabular}




\begin{tabular}{|c|c|c|c|c|}
\hline \multirow[t]{2}{*}{ Variables } & \multicolumn{2}{|c|}{ Model 1} & \multicolumn{2}{|c|}{ Model 2} \\
\hline & OR & $95 \% \mathrm{Cl}$ & OR & $95 \% \mathrm{Cl}$ \\
\hline Stroke (Yes vs. No) & & & 1.67 & $0.97-2.86$ \\
\hline Kidney disease (Yes vs. No) & & & 1.80 & $1.35-2.39$ \\
\hline Digestive disease (Yes vs. No) & & & 1.54 & $1.30-1.82$ \\
\hline Psychiatric disease (Yes vs. No) & & & 1.07 & $0.60-1.90$ \\
\hline Asthma (Yes vs. No) & & & 1.17 & $0.75-1.81$ \\
\hline
\end{tabular}

Sensitivity analysis

Firstly, we compared the differences of missing data in demographic and clinical variables between the case and control group and found no significant difference in any variable (Supplementary table 2). Secondly, during sensitivity analysis, we composed different models to estimate risk factors of symptomatic knee OA using complete raw data (i.e. complete case analysis) and multiple imputation data (Supplementary table 3, models 2 and 3). Furthermore, since the BMI group variable (categorical) had more than $20 \%$ of the values missing, while the proportions of missing values in each of the other variables were less than $5 \%$, we added one more model using complete raw data but excluded the BMI group variable (Supplementary table 3, model 4). The results from the models 3 and 4 were consistent with that those from model 2.

\section{Discussion}

Using data collected from the CHARLS, a national population survey with a 4-year follow-up, our study found that the cumulative incidence of symptomatic knee OA over 4 years among Chinese adults aged $\geq 45$ years was $8.5 \%$. Our study also showed significant variations of the incidence by province. To the best of our knowledge, this is the first study to report the incidence of symptomatic knee OA among the Chinese population. The findings may provide valuable information for health-care policy makers, allowing them to better allocate healthcare resources and develop evidence-informed health-care planning by province. In particular, the findings may have important implications for those provinces with higher incidence.

Few studies have examined incident symptomatic knee OA. In the Framingham Osteoarthritis Study, with a 8.1-year follow-up, the incidence rate of symptomatic knee OA was $6.7 \%$ ( $0.8 \%$ per year) [12]. In the current study, with a 4-year follow-up, the estimated incidence of symptomatic knee OA is $8.5 \%$ (2.1\% per year), higher than that in the Framingham Osteoarthritis Study.

Some studies have examined the incidence of radiographic knee OA. One study in the Japan showed that the incidence rate of $\mathrm{K} / \mathrm{L}$ grade $\geq 2$ knee OA was $2.9 \%$ per year [10]. Another study in the U.K. showed that the incident rate of $\mathrm{K} / \mathrm{L}$ grade $\geq 2$ knee $0 \mathrm{~A}$ was $2.5 \%$ per year [11]. However, a study in the Spain showed that the incidence rate of knee OA was identified using International Classification of Diseases (ICD)-10 codes, was $0.64 \%$ per year [13]. However, the definitions of the cases varied among these studies.

Other longitudinal studies conducted in developed countries reported risk factors including gender, age, occupation, BMI, education, household income, diabetes mellitus, hypertension, cardiovascular disease (CVD), and physical activity [5, 10, 19-22]. Our study also identified a number of risk factors associated with the development of symptomatic knee OA, including the female gender, residing in a rural area or West China, a lower level of education, lack of physical activities, hypertension, heart disease, kidney disease, and digestive disease. However, the findings should be interpreted with caution, as the causal pathways of these factors have not yet been clearly understood. The reported results about risk factors may represent total or direct effect, depending on the causal relation among the risk factors [23].

The incidence of symptomatic knee OA was significantly higher in women, consistent with previous studies $[10,13,24]$. This is likely due to women doing more household work, having a higher awareness of knee OA, or having less muscle strength and low bone mineral density. Consistent with other studies, our analysis found that senior ages were associated with higher risk of symptomatic knee OA [3, 10, $13,25]$. In addition, our study found that the incidence was highest among respondents aged 60-69 years, then decreased after 70 years, possibly because elderly persons generally do not do heavy physical work after the age of 70 years; thus, have less load on their knees. Our study also found that those with a higher level of education had a lower risk of symptomatic knee OA, consistent with previous studies $[9,26]$. This is likely due to those receiving less education being more likely to be employment in physical labor. 
In our study, those residing in rural areas or in West part of China had a higher risk of symptomatic knee OA. Meanwhile, the provinces with the highest incidence were mainly in the West region. These findings were consistent with some previous cross-sectional results [9, 25, 27]. This may be because residents in rural areas often have less-privileged socioeconomic conditions and limited access to health-care resources, while undertaking more physical labor. The difference among the three regions is also attributable to the difference in terrain and socioeconomic imbalance.

We found that people doing some sort of physical activities (e.g. dancing, body building) often had lower risk of symptomatic knee OA, which is similar to the findings of a review about OA [28]. As shown earlier, a light and moderate level of activity may be associated with less subsequent disabilities, such as knee OA [29]. This finding suggested that regular physical activity is always warranted for preventing the development of this condition.

Self-reported hypertension, heart disease, kidney disease, and digestive disease are associated with symptomatic knee OA, as shown by our study. One set of meta-analysis results showed that hypertension was significantly associated with higher symptomatic knee OA [21]. One possible explanation is that they share traditional risk factors, such as chronic inflammation. One study confirmed that CVD was a risk factor for knee $\mathrm{OA}^{15}$. A higher risk of CVD has also been observed in people with OA [30]. For these analyses, CVD included heart disease. It is possible that heart disease and symptomatic OA have a bidirectional relationship with OA. Similarly, self-reported kidney disease and digestive disease were associated with symptomatic knee OA is possible that kidney disease and digestive disease may be caused by symptomatic knee OA. The chronic inflammation and nonsteroidal anti-inflammatory drug (NSAID) treatment in symptomatic knee OA patients are reported to increase the risk of getting kidney disease and digestive disease [31, 32]. Further studies are warranted to confirm the relationship between these diseases and knee OA. Another possible explanation might be that persons with these diseases have more contact with health care and, thus, are more prone to receive a diagnosis of arthritis.

Our study has several strengths. Firstly, the CHARLS included a nationwide representative sample of middle-aged and older adults. The findings are generalizable to the Chinese population. Secondly, the survey was conducted using a strict quality-control program, and the study participants were chosen according to a strict multistage probability sampling procedure. Finally, we have reported both the incidence and associated risk factors for symptomatic knee OA, which is helpful for healthcare policy development and clinical practice.

Our study has some limitations. Firstly, the respondents in the CHARLS did not undergo radiographic assessment, the diagnosis of symptomatic knee OA was based on self-reported knee pain and self-reported arthritis diagnosis by a physician, which differed from other studies using recommendations for diagnosis of knee OA [33]. However, this definition has been used in published studies [9, 34]. Secondly, the data for other chronic diseases were based on self-reporting. Hence, the associations we observed might be affected by people with these diseases having more contact with health care and, thus, being more prone to receive a diagnosis of arthritis. However, our findings were generally consistent with previous studies $[15,21]$. Finally, findings regarding risk factors should be interpreted with caution since different definition of knee OA have different risk factors, and our findings may differ from other studies focusing on other diagnosis of knee OA.

\section{Conclusions}

Using data from the CHARLS, we observed that the cumulative incidence of symptomatic knee OA among middle-aged or older Chinese adults was high, was even more common among females, and varied by province and region. Those adults not undertaking physical activities, or presenting with heart disease, kidney disease, or digestive diseases had a higher likelihood of developing symptomatic knee OA. We believe that the findings are intriguing for health care, and have important implications for practitioners and policy makers, particularly those from developing countries.

\section{Abbreviations}

OA: osteoarthritis; CHARLS: China Health and Retirement Longitudinal Study; PPS: probability-proportional-to-size; GDP: gross domestic product; PSUs: primary sampling units; BMI: body mass index; WHO: World Health Organization.

\section{Declarations}

\section{Ethics Approval and Consent to Participate}

The current study is a secondary analysis of the CHARLS public data. The CHARLS was approved by the Ethical Review Committee of Peking University, and all participants signed informed consent at the time of participation. No separate ethical approval was required for 
our study.

\section{Consent for publication}

The current study is a secondary analysis of the CHARLS public data. No separate consent for publication was required for our study.

Availability of data and supporting materials section.

All data collected in the CHARLS are maintained at the National School of Development of Peking University, Beijing, China. The datasets are available from http://charls.pku.edu.cn/pages/data/111/zh-cn.html.

\section{Acknowledgements}

The authors thank all workers who collected the data.

\section{Funding Information}

This research was supported by National Key R\&D Program of China (Grant No. 2017YFC1700406) and 1.3.5 project for disciplines of excellence, West China Hospital, Sichuan University (Grant No. ZYYC08003).

\section{Author Contributions}

All authors were involved in drafting the article or revising it critically for important intellectual content, and all authors approved the final version to be published. Dr. Ren had full access to all of the data in the study and takes responsibility for the integrity of the data and the accuracy of the data analysis.

Study conception and design. Ren, Sun, Tan, Hu, Tang.

Acquisition of data. Ren, Li, Yang, Liu, He, Zou.

Analysis and interpretation of data. Ren, Sun, Tan, Hu, Tang.

\section{Competing Interests}

The authors declare no competing interests.

\section{References}

1. Wu YS, Dang JW. China Report of the Development on Siliver Industry (2014). Social Sciences Academic Press(China). 2014.

2. Campisi J, Kapahi P, Lithgow GJ, Melov S, Newman JC, Verdin E. From discoveries in ageing research to therapeutics for healthy ageing. Nature. 2019;571: 183-92.

3. Cross M, Smith E, Hoy D, Nolte S, Ackerman I, Fransen M, et al. The global burden of hip and knee osteoarthritis: estimates from the global burden of disease 2010 study. Annals of the rheumatic diseases. 2014;73: 1323-30.

4. Oliveria SA, Felson DT, Reed JI, Cirillo PA, Walker AM. Incidence of symptomatic hand, hip, and knee osteoarthritis among patients in a health maintenance organization. Arthritis and rheumatism. 1995;38:1134-41.

5. Silverwood V, Blagojevic-Bucknall M, Jinks C, Jordan JL, Protheroe J, Jordan KP. Current evidence on risk factors for knee osteoarthritis in older adults: a systematic review and meta-analysis. Osteoarthritis and cartilage. 2015;23:507-15.

6. Chung M, Zhao N, Wong JB, Wang C. Prospective economic evaluation of the costs for medications and nutraceuticals in knee osteoarthritis. Osteoarthritis \& Cartilage. 2016;24:S349-S50.

7. Loza E, Lopez-Gomez JM, Abasolo L, Maese J, Carmona L, Batlle-Gualda E, et al. Economic burden of knee and hip osteoarthritis in spain. Arthritis care \& research. 2010;61:158-65.

8. Kim S. Changes in surgical loads and economic burden of hip and knee replacements in the US: 1997-2004. Arthritis Rheum. 2010;59:481-8.

9. Tang X, Wang SF, Zhan SY, Niu JB, Tao K, Zhang YQ, et al. The Prevalence of Symptomatic Knee Osteoarthritis in China Results From the China Health and Retirement Longitudinal Study. Arthritis \& Rheumatology. 2016;68:648-53. 
10. Muraki S, Akune T, Oka H, Ishimoto Y, Nagata K, Yoshida M, et al. Incidence and risk factors for radiographic knee osteoarthritis and knee pain in Japanese men and women: a longitudinal population-based cohort study. Arthritis Rheum. 2012;64:1447-56.

11. Cooper C, Snow S, Mcalindon TE, Kellingray S, Stuart B, Coggon D, et al. Risk factors for the incidence and progression of radiographic knee osteoarthritis. Arthritis \& Rheumatism. 2000;43:995-1000.

12. Felson DT, Zhang Y, Hannan MT, Naimark A, Weissman BN, Aliabadi P, et al. The incidence and natural history of knee osteoarthritis in the elderly. The Framingham Osteoarthritis Study. Arthritis \& Rheumatism. 1995;38:1500-5.

13. Prieto-Alhambra D, Judge A, Javaid MK, Cooper C, Diez-Perez A, Arden NK. Incidence and risk factors for clinically diagnosed knee, hip and hand osteoarthritis: influences of age, gender and osteoarthritis affecting other joints. Annals of the rheumatic diseases. 2014;73:1659-64.

14. Xiang ZY, Mao JC, Huan-Ru QU, Xian-Guo XU, Xiang-Feng XU, Jin-Song HU, et al. Epidemiological study on risk factors of knee osteoarthritis in Shanggang Community in Pudong New District. Journal of Shanghai Jiaotong University. 2013;33:318-22.

15. Zhang J, Song L, Liu G, Zhang A, Dong H, Liu Z, et al. Risk factors for and prevalence of knee osteoarthritis in the rural areas of Shanxi Province, North China: a COPCORD study. Rheumatology International. 2013;33:2783-8.

16. Jiang L, Rong J, Zhang Q, Hu F, Zhang S, Li X, et al. Prevalence and associated factors of knee osteoarthritis in a community-based population in Heilongjiang, Northeast China. Rheumatology International. 2012;32:1189-95.

17. Du H, Chen SL, Bao CD, Wang XD, Lu Y, Gu YY, et al. Prevalence and risk factors of knee osteoarthritis in Huang-Pu District, Shanghai, China. Rheumatology International. 2005;25:585-90.

18. Zhao Y, Hu Y, Smith JP, Strauss J, Yang G. Cohort profile: the China Health and Retirement Longitudinal Study (CHARLS). Int J Epidemiol. 2014;43:61-8.

19. Louati K, Vidal C, Berenbaum F, Sellam J. Association between diabetes mellitus and osteoarthritis: systematic literature review and meta-analysis. RMD open. 2015;1:e000077.

20. Mcwilliams DF, Leeb BF, Muthuri SG, Doherty M, Zhang W. Occupational risk factors for osteoarthritis of the knee: a meta-analysis. Osteoarthritis \& Cartilage. 2011;19:829-39.

21. Zhang YM, Wang J, Liu XG. Association between hypertension and risk of knee osteoarthritis: A meta-analysis of observational studies. Medicine. 2017;96:e7584.

22. Nelson AE. Osteoarthritis year in review 2017: clinical. Osteoarthritis and cartilage. 2018;26:319-25.

23. Westreich D, Greenland S. The Table 2 Fallacy: Presenting and Interpreting Confounder and Modifier Coefficients. American Journal of Epidemiology. 2013;177:292-8.

24. Srikanth VK, Fryer JL, Zhai G, Winzenberg TM, Hosmer D, Jones G. A meta-analysis of sex differences prevalence, incidence and severity of osteoarthritis. Osteoarthritis and cartilage. 2005;13:769-81.

25. Liu Y, Zhang H, Liang N, Fan W, Li J, Huang Z, et al. Prevalence and associated factors of knee osteoarthritis in a rural Chinese adult population: an epidemiological survey. BMC Public Health. 2016;16.

26. Leigh JP, Fries JF. Correlations between education and arthritis in the 1971-1975 NHANES I. Social Science \& Medicine. 1994;38:57583.

27. Kang X, Fransen M, Zhang Y, Li H, Ke Y, Lu M, et al. The high prevalence of knee osteoarthritis in a rural Chinese population: The Wuchuan osteoarthritis study. Arthritis \& Rheumatism. 2009;61:641-7.

28. Sharma L. Osteoarthritis year in review 2015: clinical. Osteoarthritis and cartilage. 2016; 24: 36-48.

29. Dunlop DD, Song J, Semanik PA, Sharma L, Bathon JM, Eaton CB, et al. Relation of physical activity time to incident disability in community dwelling adults with or at risk of knee arthritis: prospective cohort study. BMJ. 2014;348:g2472.

30. Veronese N, Trevisan C, De Rui M, Bolzetta F, Maggi S, Zambon S, et al. Association of Osteoarthritis With Increased Risk of Cardiovascular Diseases in the Elderly: Findings From the Progetto Veneto Anziano Study Cohort. Arthritis \& Rheumatology. 2016;68:1136-44.

31. Nissen SE, Yeomans ND, Solomon DH, Lüscher TF, Libby P, Husni ME, et al. Cardiovascular Safety of Celecoxib, Naproxen, or Ibuprofen for Arthritis. N Engl J Med. 2016;375:2519-29.

32. da Costa BR, Reichenbach S, Keller N, Nartey L, Wandel S, Jüni P, et al. Effectiveness of non-steroidal anti-inflammatory drugs for the treatment of pain in knee and hip osteoarthritis: a network meta-analysis. Lancet. 2016;387:2093-105.

33. Zhang W, Doherty M, Peat G, Bierma-Zeinstra MA, Arden NK, Bresnihan B, et al. EULAR evidence-based recommendations for the diagnosis of knee osteoarthritis. Annals of the rheumatic diseases. 2009;69:483-9. 
34. Liu Q, Wang S, Lin J, Zhang Y. The burden for knee osteoarthritis among Chinese elderly: estimates from a national representative study. Osteoarthritis and Cartilage. 2018;26:1636-42.

\section{Figures}

17708 respondents in the 2011 national baseline survey

184 respondents did not answer both the knee pain and the physiciandiagnosis questions, 1614 respondents had symptomatic KOA at the 2011 national baseline survey.

15910 respondents were free from
symptomatic KOA at the 2011 national
baseline survey

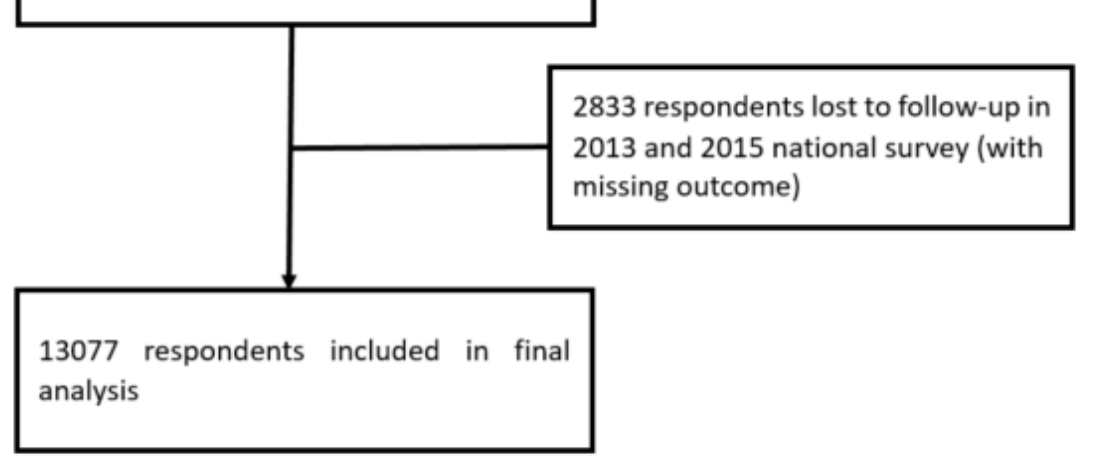

\section{Figure 1}

Flowchart showing the selection of the respondents who were included in the final analysis in this study

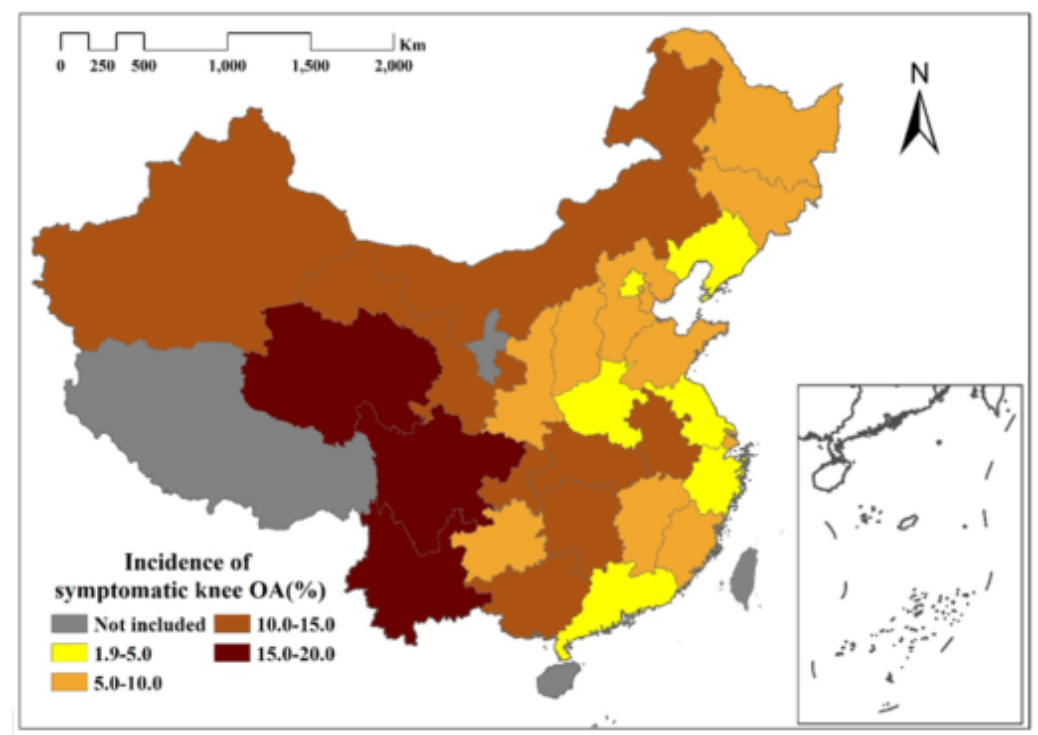

Figure 2 
Incidence of symptomatic knee OA in different provinces of China. Note: The designations employed and the presentation of the material on this map do not imply the expression of any opinion whatsoever on the part of Research Square concerning the legal status of any

country, territory, city or area or of its authorities, or concerning the delimitation of its frontiers or boundaries. This map has been provided by the authors.

\section{Supplementary Files}

This is a list of supplementary files associated with this preprint. Click to download.

- SupplementaryTable13.docx 